\title{
One-sided duo property on nilpotents
}

\author{
Chan Yong Hong ${ }^{1}$ (D), Hong Kee Kim² (D), Nam Kyun Kim³ (D), Tai Keun Kwak*4 (D), \\ Yang Lee Li $^{5}$ \\ ${ }^{1}$ Department of Mathematics, Kyung Hee University, Seoul 02447, Korea \\ ${ }^{2}$ Department of Mathematics and RINS, Gyeongsang National University, Jinju 52828, Korea \\ ${ }^{3}$ School of Basic Sciences, Hanbat National University, Daejeon 34158, Korea \\ ${ }^{4}$ Department of Mathematics, Daejin University, Pocheon 11159, Korea \\ ${ }^{5}$ Department of Mathematics, Yanbian University, Yanji 133002, China and Institute of Basic Science, \\ Daejin University, Pocheon 11159, Korea
}

\begin{abstract}
We study the structure of nilpotent elements in relation with a ring property that is near to one-sided duo rings. Such a property is said to be one-sided nilpotent-duo. We prove the following for a one-sided nilpotent-duo ring $R$ : (i) The set of nilpotents in $R$ forms a subring; (ii) Köthe's conjecture holds for $R$; (iii) the subring generated by the identity and the set of nilpotents in $R$ is a one-sided duo ring; (iv) if the polynomial ring $R[x]$ over $R$ is one-sided nilpotent-duo then the set of nilpotents in $R$ forms a commutative ring, and $R[x]$ is an NI ring. Several connections between one-sided nilpotent-duo and one-sided duo are given. The structure of one-sided nilpotent-duo rings is also studied in various situations in ring theory. Especially we investigate several kinds of conditions under which one-sided nilpotent-duo rings are NI.
\end{abstract}

Mathematics Subject Classification (2010). 16N40, 16U80

Keywords. right (left) nilpotent-duo ring, nilpotent, right (left) duo ring, radical, NI ring, matrix ring, ascending chain condition on left (right) annihilators

Throughout this paper all rings are associative with identity unless otherwise specified. Let $R$ be a ring. We use $N(R), J(R), N_{*}(R)$, and $N^{*}(R)$ to denote the set of all nilpotent elements, Jacobson radical, lower nilradical (i.e., prime radical), and upper nilradical (i.e., the sum of all nil ideals) of $R$, respectively. A nilpotent element is also called a nilpotent for simplicity. It is well-known that $N_{*}(R) \subseteq N^{*}(R) \subseteq N(R)$ and $N^{*}(R) \subseteq J(R)$. $U(R)$ denotes the group of all units in $R$. Denote the $n$ by $n$ full (resp., upper triangular) matrix ring over $R$ by $\operatorname{Mat}_{n}(R)$ (resp., $T_{n}(R)$ ). Write $D_{n}(R)=\left\{\left(a_{i j}\right) \in T_{n}(R) \mid a_{11}=\cdots=a_{n n}\right\}$ and use $E_{i j}$ for the matrix with $(i, j)$-entry 1 and elsewhere $0 . \mathbb{Z}\left(\mathbb{Z}_{n}\right)$ denotes the ring of integers (modulo $n$ ).

\footnotetext{
*Corresponding Author.

Email addresses: hcy@khu.ac.kr (C.Y. Hong), hkkim@gsnu.ac.kr (H.K. Kim), nkkim@hanbat.ac.kr (N.K. Kim), tkkwak@daejin.ac.kr (T.K. Kwak) ylee@pusan.ac.kr (Y. Lee)

Received: 23.05.2019; Accepted: 10.03.2020
} 


\section{Right (left) nilpotent-duo rings}

In this section, we observe basic properties and examples of right nilpotent-duo rings. We also analyze the connections between right nilpotent-duo rings and related rings.

A ring $R$ is usually called reduced if $N(R)=0$. Due to Bell [1], a right (or left) ideal $I$ of a ring $R$ is said to have the insertion of factors property (simply, IFP) if $a b \in I$ implies $a R b \subseteq I$ for $a, b \in R$. A ring $R$ is called IFP if the zero ideal of $R$ has the IFP. A ring is usually called abelian if every idempotent is central. Reduced rings are IFP, and IFP rings are abelian.

Following Feller [8], a ring is called right (resp., left) duo if every right (resp., left) ideal is an ideal; a ring is called duo if it is both right and left duo. It is easily shown that right (left) duo rings are IFP. For any IFP ring $R$, it is well-known that $N(R)=N_{*}(R)$. There are very useful results for one-sided duo rings in $[3,25]$.

We now consider the right duo property on the set of nilpotent elements in a ring. A ring $R$ (possibly without identity) will be called right nilpotent-duo if $N(R) a \subseteq a N(R)$ for every $a \in R$. Left nilpotent-duo rings are defined similarly. A ring will be called nilpotent$d u o$ if it is both left and right nilpotent-duo. Note that a ring $R$ is nilpotent-duo if and only if $N(R) a=a N(R)$ for each $a \in R$. It is easily checked that both commutative rings and reduced rings are nilpotent-duo. Recall that a ring $R$ satisfies Köthe's conjecture if the sum of two nil left ideals of $R$ is also nil.

Theorem 1.1. Let $R$ be a right (resp., left) nilpotent-duo ring. Then we have the following.

(1) $N(R)$ forms a subring of $R$.

(2) Köthe's conjecture holds.

(3) $N(R)$ is a right (resp., left) duo ring without identity.

(4) The subring of $R$ generated by $N(R)$ and the identity 1 of $R$ is a right (resp., left) duo ring.

Proof. (1) Let $a, b \in N(R)$. Say $a^{m}=0$ and $b^{n}=0$ for $m, n \geq 1$. First consider $(a b)^{m}$. We use the right nilpotent-duo property of $R$ freely. There exists $b_{1} \in N(R)$ such that $b a=a b_{1}$. Next $b_{1} a=a b_{2}$ for some $b_{2} \in N(R)$, and similarly $b_{2} a=a b_{3}$ for some $b_{2} \in N(R)$. Inductively, we can take $b_{i+1} \in N(R)$ such that $b_{i} a=a b_{i+1}$ for all $i \geq 1$. Then

$$
\begin{aligned}
(b a)^{m} & =\left(a b_{1}\right)^{m} \\
& =\left(a b_{1}\right)\left(a b_{1}\right)\left(a b_{1}\right)^{m-2}=a\left(b_{1} a\right) b_{1}\left(a b_{1}\right)^{m-2}=a\left(a b_{2}\right) b_{1}\left(a b_{1}\right)^{m-2}=a^{2} b_{2} b_{1}\left(a b_{1}\right)^{m-2} \\
& =a^{2} b_{2}\left[b_{1}\left(a b_{1}\right)\right]\left(a b_{1}\right)^{m-3}=a^{2} b_{2}\left(a b_{2}\right) b_{1}\left(a b_{1}\right)^{m-3} \\
& =a^{2}\left(a b_{3}\right) b_{2} b_{1}\left(a b_{1}\right)^{m-3}=a^{3} b_{3} b_{2} b_{1}\left(a b_{1}\right)^{m-3} \\
& =\cdots \\
& =a^{m} b_{m} b_{m-1} \cdots b_{2} b_{1}=0 .
\end{aligned}
$$

Thus $b a, a b \in N(R)$. As a consequence, we obtain that

$$
e_{1} e_{2} \cdots e_{s+t}=a^{s} c \text { and } e_{1} e_{2} \cdots e_{s+t}=b^{t} d \text { with } c, d \in N(R),
$$

where $s, t \geq 1, e_{i}$ is either $a$ or $b$, and $\left|\left\{e_{i} \mid e_{i}=a\right\}\right|=s,\left|\left\{e_{i} \mid e_{i}=b\right\}\right|=t$, where $|S|$ denotes the cardinality of given a set $S$.

Next we shall prove $a+b \in N(R)$. Let $l=\max \{m, n\}$, and consider $(a+b)^{2 l}$. Then every term of the expansion of $(a+b)^{2 l}$ is of the form

$$
a^{l} f \text { or } b^{l} g \text { with } f, g \in N(R) .
$$

But $a^{l}=0$ and $b^{l}=0$; hence $(a+b)^{2 l}=0$. This implies $a+b \in N(R)$, and therefore $N(R)$ forms a subring of $R$. The proof for the left case is similar. 
(2) This is an immediate consequence of (1).

(3) Note that $N(R) a \subseteq a N(R)$ for all $a \in N(R)$. Moreover $N(R)$ forms a subring of $R$ by (1); hence both $N(R) a$ and $a N(R)$ are contained in $N(R)$. Thus $N(R)$ is a right duo ring. The left case is similarly proved.

(4) Let $S$ be the subring of $R$ generated by 1 and $N(R)$. Since $\mathbb{Z} \cdot 1$ is contained in the center of $R, S=\mathbb{Z} \cdot 1+N(R)$ by (1), where $\mathbb{Z} \cdot 1=\{n 1 \mid n \in \mathbb{Z}\}$. Note $N(\mathbb{Z} \cdot 1) \subseteq N(R)$ and $N(S)=N(\mathbb{Z} \cdot 1)+N(R)=N(R)$. Let $a \in S$ and $d=m 1+c \in S$ with $m \in \mathbb{Z}$ and $c \in N(R)$. Since $R$ is right nilpotent-duo, $c a=a c_{1}$ for some $c_{1} \in N(R)$. Thus we get

$$
d a=(m 1+c) a=(m 1) a+c a=a(m 1)+a c_{1}=a\left(m 1+c_{1}\right) .
$$

But $c_{1} \in N(R)=N(S)$ and $m 1+c_{1} \in S$ follows. Therefore $S$ is right duo. The proof for the left case is similar.

In the following we see a connection between right duo rings and right nilpotent-duo rings via a sort of matrix ring. $C(R)$ denotes the monoid of all regular elements (i.e., non-zero-divisors) in a ring $R$.

Theorem 1.2. Let $R$ be a ring. Then we have the following.

(1) If $D_{2}(R)$ is right duo (resp., right nilpotent-duo), then for any $a \in R$ (resp., $a \in$ $N(R))$ and $b \in R$ we have $a b-b a \in b^{2} R$.

(2) (i) Let $R$ be a right (resp., left) duo ring such that $R \backslash C(R)=N(R)$. Then $R$ is right (resp., left) nilpotent-duo.

(ii) Let $R$ be a right (resp., left) duo ring that is local and $J(R)=N(R)$. Then $R$ is right (resp., left) nilpotent-duo.

(iii) Let $R$ be a right (resp., left) duo ring. Then $N(R) a \subseteq a N(R)$ (resp., $a N(R) \subseteq$ $N(R)$ a) for $a \in C(R)$.

(3) If $D_{2}(R)$ is right (resp., left) nilpotent-duo, then $R$ is right (resp. left) nilpotent-duo and right (resp., left) duo.

(4) If every nilpotent of $R$ is central and $R$ is right (resp., left) duo, then $D_{2}(R)$ is right (resp.,left) nilpotent-duo.

(5) Let $R$ be a reduced ring. Then $R$ is right (resp., left) duo if and only if $D_{2}(R)$ is right (resp., left) nilpotent-duo.

Proof. (1) For any $a \in R$ (resp., $a \in N(R))$ and $b \in R$, there exist $c \in R$ (resp., $c \in N(R)$ ) and $d \in R$ such that $\left(\begin{array}{ll}a & 0 \\ 0 & a\end{array}\right)\left(\begin{array}{ll}b & 1 \\ 0 & b\end{array}\right)=\left(\begin{array}{ll}b & 1 \\ 0 & b\end{array}\right)\left(\begin{array}{ll}c & d \\ 0 & c\end{array}\right)$, i.e., $a b=b c$ and $a=b d+c$. Hence $a b-b a=b c-b(b d+c)=-b^{2} d \in b^{2} R$.

(2) (i) Let $b \in N(R)$ with $b^{n}=0$ for $n \geq 2$ and $0 \neq a \in R$. Since $R$ is right duo, $b a=a c$ for some $c \in R$. Then $0=b^{n} a=b^{n-1} a c=b^{n-2} b a c=b^{n-2} a c^{2}=\cdots=a c^{n}$. Assume $c \in C(R)$, then $a=0$, contrary to $a \neq 0$. So $c \notin C(R)$, so that $c \in N(R)$ by hypothesis. Thus $R$ is right nilpotent-duo. The proof for the left case is done by symmetry.

(ii) This is clear from (i).

(iii) Let $b \in N(R)$ with $b^{n}=0$ for $n \geq 2$ and $a \in C(R)$. Then, by the proof of (i), we get $b a=a c$ and $a c^{n}=0$ for some $c \in R$. Since $a \in C(R)$, we have $c^{n}=0$. This concludes $N(R) a \subseteq a N(R)$. The proof for the left case is done by symmetry.

(3) Let $E=D_{2}(R)$. First note that $N(E)=\left\{\left(\begin{array}{ll}a & b \\ 0 & a\end{array}\right) \mid a \in N(R), b \in R\right\}$. Suppose that $E$ is right nilpotent-duo and let $x, y \in R$. Since $\left(\begin{array}{ll}0 & x \\ 0 & 0\end{array}\right) \in N(E),\left(\begin{array}{ll}0 & x \\ 0 & 0\end{array}\right)\left(\begin{array}{ll}y & 0 \\ 0 & y\end{array}\right)=$ $\left(\begin{array}{ll}y & 0 \\ 0 & y\end{array}\right)\left(\begin{array}{ll}z & u \\ 0 & z\end{array}\right)$ for some $\left(\begin{array}{cc}z & u \\ 0 & z\end{array}\right) \in N(E)$. This yields $x y=y u$, hence $R$ is right duo. 
Next let $a \in N(R)$ to show $R$ being right nilpotent duo. Then $c \in N(R)$ by the proof of (1). Since $R$ is right duo, we have $N(R)=N_{*}(R)$. So $b d=a-c \in N(R)$ and then $a b=b a-b^{2} d=b(a-b d) \in b N(R)$. Hence $R$ is right nilpotent-duo. The proof for the left case can be done similarly.

(4) Suppose that every nilpotent of $R$ is central and $R$ is right duo. Let $A=\left(\begin{array}{ll}a & b \\ 0 & a\end{array}\right), B=$ $\left(\begin{array}{ll}c & d \\ 0 & c\end{array}\right) \in E$ with $A \in N(E)$. Then $a \in N(R)$ and so $a$ is central by hypothesis. Since $R$ is right duo, $b c=c b^{\prime}$ for some $b^{\prime} \in R$. Now letting $A^{\prime}=\left(\begin{array}{ll}a & b^{\prime} \\ 0 & a\end{array}\right)$, we get $A^{\prime} \in N(E)$ and $A B=\left(\begin{array}{cc}a c & a d+b c \\ 0 & a c\end{array}\right)=\left(\begin{array}{cc}c a & d a+c b^{\prime} \\ 0 & c a\end{array}\right)=B A^{\prime}$. Thus $E$ is right nilpotent-duo. The proof for the left case is similar.

(5) is an immediate consequence of $(3,4)$.

As in Theorem 1.2(2), we have a partial argument for right duo rings to be right nilpotent-duo. So we raise the following.

Questions. (1) Can we delete the condition " $R \backslash C(R)=N(R)$ " in Theorem 1.2(2)(i)?

(2) Let $R$ be a right duo ring. Then $N(R) a \subseteq a N(R)$ for $a \notin C(R)$ ?

The condition " $R$ is a reduced ring" in Theorem 1.2(5) is not superfluous by the example below.

Example 1.3. There exists a non-reduced right duo ring $R$ over which $D_{2}(R)$ is not right nilpotent-duo. We refer to the construction and argument of Courter in [6, Example 3.4]. Let $K$ be a field and $E$ be the row finite infinite matrix ring over $K$. Let $S$ be the $K$-subspace of $E$ generated by the matrices $\left\{a_{0}, a_{1}, \ldots, a_{j}, \ldots\right\}$, where

$$
a_{0}=E_{12}, a_{1}=E_{13}, a_{2}=E_{14}+E_{32}, \ldots, a_{j}=E_{1(j+2)}+E_{(j+1) 2} \text { for } j \geq 2 .
$$

Next let $I$ be the identity matrix in $E$ and $R=K I \oplus S$, i.e., $R$ is the $K$-subspace of $E$ with a basis $\left\{I, a_{0}, a_{1}, \ldots, a_{j}, \ldots\right\}$. Then $R$ is a right duo ring by [6, Example 3.4], but non-reduced as can be seen by $E_{12}^{2}=0$. Every element in $R$ is expressed by $k I+\sum_{i=0}^{m} c_{i} a_{i}$ with $m \geq 1$ and $k, c_{0}, \ldots, c_{m} \in K$. Moreover $S a_{0}=0=a_{0} S, a_{i} a_{i+1}=a_{0}$ for $i \geq 1$, and $a_{i} S=K a_{0}$. So $R$ is a local ring such that $J(R)=N(R)=N^{*}(R)=N_{*}(R)=S$, $J(R)^{2}=K a_{0}$, and $J(R)^{3}=0$.

Moreover $a_{1} \in N(R)$ and $a_{1} a_{2}-a_{2} a_{1}=a_{0} \notin a_{2}^{2} R=0$, it immediately follows from Theorem 1.2(1) that $D_{2}(R)$ is neither right nilpotent-duo nor right duo. But $R$ is right duo and local with $J(R)=N(R)$, and so $R$ is right nilpotent-duo by Theorem 1.2(2). This also shows that $D_{2}(R)$ over a right nilpotent-duo ring $R$ need not be right nilpotent-duo.

Considering Theorem 1.2(5), it is natural to argue about the nilpotent-duo property of $D_{n}(R)$ for the case of $n \geq 3$, where $R$ is a reduced ring. But we have a negative result as follows.

Example 1.4. Let $R$ be any reduced ring and consider $E=D_{n}(R)$ for $n \geq 3$. Then $N(E)=\left\{\left(a_{i j}\right) \in E \mid a_{11}=\cdots=a_{n n}=0\right\}$.

Consider the subset $E_{12} N(E)=R E_{13}+\cdots+R E_{1 n}$. But $N(E) E_{12}=0$ and so $E_{12} N(E)$ cannot be contained in $N(E) E_{12}$. Thus $E$ is not left nilpotent-duo.

Next consider the subset $N(E) E_{(n-1) n}=R E_{1 n}+\cdots+R E_{(n-1) n}$. But $E_{(n-1) n} N(E)=0$ and so $N(E) E_{(n-1) n}$ cannot be contained in $E_{(n-1) n} N(E)$. Thus $E$ is not right nilpotentduo. 
Note that the right nilpotent-duo ring $R$ in Example 1.3 is not left nilpotent-duo because $a_{2} \in N(R)$ and $a_{1} a_{2}=a_{0} \notin R a_{1}$. We see an application of Theorem 1.2(5) in the following which also shows that the nilpotent-duo property is not left-right symmetric.

Example 1.5. (1) There is a left nilpotent-duo ring that is not right nilpotent-duo. We apply the argument in [5, Lemma 1.3(3)]. Let $S=F(t)$ be the quotient field of the polynomial ring $F[t]$ with an indeterminate $t$ over a field $F$, and $\sigma: S \rightarrow S$ be a monomorphism defined by $\sigma\left(\frac{f(t)}{g(t)}\right)=\frac{f\left(t^{2}\right)}{g\left(t^{2}\right)}$. Consider the skew power series ring $R=$ $S[[x ; \sigma]]$ in which every element is of the form $\sum_{i=0}^{\infty} a_{i} x^{i}$, only subject to $x a=\sigma(a) x$ for $a \in S$. Let $E=D_{2}(R)$. Then

$$
\begin{gathered}
U(E)=\left\{\left(\begin{array}{cc}
f(x) & r(x) \\
0 & f(x)
\end{array}\right) \in E \mid \text { the constant term of } f(x) \text { is nonzero and } r(x) \in R\right\} \text { and } \\
N(E)=\left\{\left(\begin{array}{cc}
0 & s(x) \\
0 & 0
\end{array}\right) \in E \mid s(x) \in R\right\} .
\end{gathered}
$$

Let $0 \neq f(x) \in R$. Then we can write $f(x)=a_{0} x^{k}+a_{1} x^{k+1}+\cdots \in R$ with $a_{0} \neq 0$ and $k \geq 0$. Write $h(x)=\sum_{i=0}^{\infty} a_{i} x^{i}$. Then $f(x)=h(x) x^{k}$ and $h(x) \in U(R)$. Let $g(x)=t^{2 l+1}+\sum_{j=1}^{\infty} b_{j} x^{j} \in R$ with $l \geq 0$. Then there cannot exist $k(x) \in R$ such that $g(x) f(x)=f(x) k(x)$ by the argument in [5, Lemma 1.3(3)].

Let $A=\left(\begin{array}{cc}f(x) & f_{1}(x) \\ 0 & f(x)\end{array}\right)$ with $f_{1}(x) \in R$ and $B=\left(\begin{array}{cc}0 & g(x) \\ 0 & 0\end{array}\right)$. Assume that $E$ is right nilpotent duo. Hence there exists $C \in N(E)$ such that $B A=A C$. Then we can write $C=\left(\begin{array}{cc}0 & k(x) \\ 0 & 0\end{array}\right)$ with $k(x) \in R$ such that

$$
\left(\begin{array}{cc}
0 & g(x) \\
0 & 0
\end{array}\right)\left(\begin{array}{cc}
f(x) & f_{1}(x) \\
0 & f(x)
\end{array}\right)=\left(\begin{array}{cc}
0 & g(x) f(x) \\
0 & 0
\end{array}\right)=B A=A C=\left(\begin{array}{cc}
0 & f(x) k(x) \\
0 & 0
\end{array}\right) .
$$

This entails $g(x) f(x)=f(x) k(x)$, contrary to the argument above. Thus $E$ is not right nilpotent-duo. So $R$ is not right duo by Theorem 1.2(4), since every nilpotent of $R$ is central.

Next we claim that $E$ is left nilpotent-duo. Consider $A B$ and apply the argument in [5, Lemma 1.3(3)], where $g(x)=\sum_{j=0}^{\infty} b_{j} x^{j}$ is assumed to be any power series in $R$. Then $f(x) g(x)=h(x) x^{k} g(x)=h(x) g_{1}(x) x^{k}=h(x) g_{1}(x) h(x)^{-1} h(x) x^{k}=h(x) g_{1}(x) h(x)^{-1} f(x)$, where $x^{k} g(x)=g_{1}(x) x^{k}$ and $g_{1}(x)=\sum_{j=0}^{\infty} \sigma^{k}\left(b_{j}\right) x^{j}$. This yields

$$
A B=\left(\begin{array}{cc}
f(x) & f_{1}(x) \\
0 & f(x)
\end{array}\right)\left(\begin{array}{cc}
0 & g(x) \\
0 & 0
\end{array}\right)=\left(\begin{array}{cc}
0 & f(x) g(x) \\
0 & 0
\end{array}\right)=\left(\begin{array}{cc}
0 & h(x) g_{1}(x) h(x)^{-1} f(x) \\
0 & 0
\end{array}\right) .
$$

Here let $m(x)=h(x) g_{1}(x) h(x)^{-1}$. Then $\left(\begin{array}{cc}0 & m(x) \\ 0 & 0\end{array}\right) \in N(E), C$ say. These arguments give us $A B=C A$, and thus $E$ is left nilpotent-duo. So $R$ is left duo by Theorem 1.2(3).

(2) The ring $R$ in Example 1.3 is right nilpotent-duo. But since $a_{2} \in N(R)$ and $a_{1} a_{2}=$ $a_{0} \notin R a_{1}=K a_{1}, R$ is not left nilpotent-duo. The opposite construction, in the column finite infinite matrix ring over $K$, provides a left nilpotent-duo ring but not right nilpotentduo.

The monomorphism $\sigma$ in Example 1.5(1) is not surjective. But if $\sigma$ is surjective, then the ring $D_{2}(R)$ is nilpotent-duo as we see in the following.

Proposition 1.6. Let $K$ be a field and $\sigma$ be a monomorphism of $K$. Consider the skew power series ring $R=K[[x ; \sigma]]$ by $\sigma$ with an indeterminate $x$ over $K$, where every element is of $R$ is of the form $\sum_{i=0}^{\infty} a_{i} x^{i}$, only subject to $x a=\sigma(a) x$ for all $a \in K$. If $\sigma$ is bijective, then $D_{2}(R)$ is a nilpotent-duo ring. 
Proof. $R=K[[x ; \sigma]]$ is clearly reduced since $R$ is a domain and $\sigma$ is injective. Moreover if $\sigma$ is bijective, then $R$ is duo by applying the method in [16, Example 1]. Hence $D_{2}(R)$ is nilpotent-duo by Theorem 1.2(5).

The following example shows that nilpotent-duo rings need not be one-sided duo.

Example 1.7. (1) Any reduced ring, which is not one-sided duo, is such a ring as desired.

(i) Let $R$ be a right (resp., left) primitive domain that is not a division ring (e.g., the first Weyl algebra over a field of characteristic zero). It is well-known that every right (resp., left) primitive factor ring of a right (resp., left) duo ring is a division ring. So $R$ cannot be right (resp., left) duo, but $R$ is clearly nilpotent-duo.

(ii) $D[x]$ over a noncommutative division ring $D$ is neither right nor left duo by $[20$, Theorem 1].

(2) Let $K$ be a field of characteristic zero and $K\langle x, y\rangle$ be the free algebra with noncommuting indeterminates $x, y$ over $K$. The factor ring $R_{0}=K\langle x, y\rangle /(y x-x y-1)$ is isomorphic to the first Weyl algebra over $K$, where $(y x-x y-1)$ is the ideal of $K\langle x, y\rangle$ generated by $y x-x y-1$. Refer to [21, Theorem 1.3.5, Corollary 2.1.14, and Theorem 2.1.15] for more details. Let $R_{1}$ be the division ring of right quotients of $R_{0}$. Define

$$
R=\left\{\left(\begin{array}{cc}
a & b \\
0 & a
\end{array}\right) \mid a \in R_{0} \text { and } b \in R_{1}\right\} .
$$

Let $M_{1}=\left(\begin{array}{cc}\bar{x} & 0 \\ 0 & \bar{x}\end{array}\right), M_{2}=\left(\begin{array}{cc}\bar{y} & 0 \\ 0 & \bar{y}\end{array}\right) \in R$. Then $R M_{1}$ (resp., $M_{1} R$ ) is not a right ideal (resp., not a left ideal) of $R$ because $M_{1} M_{2} \notin R M_{1}$ (resp., $M_{2} M_{1} \notin M_{1} R$ ), noting $\bar{x} \bar{y} \notin R_{0} \bar{x}$ (resp., $\bar{y} \bar{x} \notin \bar{x} R_{0}$ ). So $R$ is neither left nor right duo.

We next claim that $R$ is nilpotent-duo. Note that $N(R)=\left(\begin{array}{cc}0 & R_{1} \\ 0 & 0\end{array}\right)$. Since $R_{1} b=$ $R_{1}=b R_{1}$ for all $0 \neq b \in R_{1}$, we have $N(R) M=M N(R)$ for all $M \in R$, noting that $N(R) M=N(R)=M N(R)$ when $M \notin N(R)$ and $N(R) M=0=M N(R)$ when $M \in N(R)$. Thus $R$ is nilpotent-duo.

The following contains basic facts about right nilpotent-duo rings with identity.

Lemma 1.8. (1) Right (left) nilpotent-duo rings are abelian.

(2) Every right Artinian, right (left) nilpotent-duo simple ring is a division ring.

(3) Let $R$ be a local ring such that $J(R)^{2}=0$. Then $R$ is nilpotent-duo.

(4) Let $I$ be a nil ideal of a ring $R$. If $R$ is a right (resp., left) nilpotent-duo ring, then $R / I$ is a right (resp., left) nilpotent-duo ring.

Proof. (1) Let $R$ be a right nilpotent-duo ring and assume on the contrary that there exist $r, e^{2}=e \in R$ with $\operatorname{er}(1-e) \neq 0$. Then $\operatorname{er}(1-e) \in N(R)$. Set $x=\operatorname{er}(1-e)$. Since $R$ is right nilpotent-duo, $x(1-e)=(1-e) y$ for some $y \in N(R)$. But

$$
0 \neq x=e(e r(1-e))(1-e)=e x(1-e)=e(1-e) y=0,
$$

a contradiction. Thus $R$ is abelian. The proof of the left case is similar.

(2) It is an immediate consequence of (1).

(3) First note $J(R)=N(R)$ because $J(R)$ is nilpotent, and so $N(R)^{2}=0$. Let $a \in R$ and $b \in N(R)$. If $a \in N(R)$ then $a b=0=b a$. Let $a \notin N(R)$. Then $a \in U(R)$ because $R / N(R)$ is a division ring. So we have $b a=a a^{-1} b a$ and $a b=a b a^{-1} a$. But $a^{-1} b a, a b a^{-1} \in N(R)$. Thus $R$ is nilpotent-duo.

(4) Let $I$ be a nil ideal of $R$. Then $N(R / I)=\{a+I \mid a \in N(R)\}$. Suppose that $R$ is right nilpotent-duo. For any $n+I \in N(R / I)$ and $a+I \in R / I,(n+I)(a+I)=n a+I=$ $a c+I \in(a+I) N(R / I)$ for some $c \in N(R)$ because $R$ is right nilpotent-duo and $n \in N(R)$. Thus $R / I$ is right nilpotent-duo. The proof for the left case is similar. 
REMARKs. (1) $\operatorname{Mat}_{n}(R)$ and $T_{n}(R)$ for all $n \geq 2$ over any ring $R$ are not abelian, and hence they are neither left nor right nilpotent-duo by Lemma 1.8(1). So the concept of right (left) nilpotent-duo ring is not Morita invariant. Moreover, $N\left(T_{n}\left(\mathbb{Z}_{2}\right)\right)$ forms a subring of $T_{n}\left(\mathbb{Z}_{2}\right)$ for all $n \geq 2$ by help of [15, Proposition 4.1(1)]; hence this fact entails that the converse of Theorem 1.1(1) need not hold.

(2) Example 1.5(1) also shows that the converse of Lemma 1.8(1) need not be true. Indeed, the ring $R$ in Example 1.5(1) is a domain, and hence $E=D_{2}(R)$ is abelian by [13, Lemma 2].

(3) Related to Lemma 1.8(1), there exists a right nilpotent-duo ring without identity as well as a right duo ring which is not abelian. Let $R=\left(\begin{array}{cc}\mathbb{Z}_{2} & \mathbb{Z}_{2} \\ 0 & 0\end{array}\right)$. Then $N(R)=\left(\begin{array}{cc}0 & \mathbb{Z}_{2} \\ 0 & 0\end{array}\right)$, $N(R)^{2}=0$, and $N(R) a=0$ for all $a \in R$. Thus $R$ is a right nilpotent-duo ring without identity. But $R$ is not left nilpotent-duo because $N(R) E_{11}=0$ cannot contain $E_{11} N(R)=$ $N(R)$.

Note that all right ideals of $R$ are $\left(\begin{array}{ll}0 & 0 \\ 0 & 0\end{array}\right), R,\left(\begin{array}{cc}0 & \mathbb{Z}_{2} \\ 0 & 0\end{array}\right)$; all left ideals are $\left(\begin{array}{ll}0 & 0 \\ 0 & 0\end{array}\right), R$, $\left(\begin{array}{cc}\mathbb{Z}_{2} & 0 \\ 0 & 0\end{array}\right),\left(\begin{array}{cc}0 & \mathbb{Z}_{2} \\ 0 & 0\end{array}\right),\left\{\left(\begin{array}{ll}0 & 0 \\ 0 & 0\end{array}\right),\left(\begin{array}{ll}1 & 1 \\ 0 & 0\end{array}\right)\right\}$; and all ideals are $\left(\begin{array}{ll}0 & 0 \\ 0 & 0\end{array}\right), R,\left(\begin{array}{cc}0 & \mathbb{Z}_{2} \\ 0 & 0\end{array}\right)$. Therefore $R$ is a right duo ring without identity, but not left duo by the existence of the left ideal $\left(\begin{array}{cc}\mathbb{Z}_{2} & 0 \\ 0 & 0\end{array}\right)$ that is not two-sided, recalling that right duo rings are abelian.

(4) Notice that if a ring $R$ is right (resp., left) nilpotent-duo then $R / N^{*}(R)$ is right (resp., left) nilpotent-duo by Lemma 1.8(4).

The class of (one-sided) nilpotent-duo rings is not closed under subrings and homomorphic images as we see in the following.

Example 1.9. (1) The class of right (left) nilpotent-duo rings is not closed under subrings:

(i) The ring $R$ in Example 1.3 is right nilpotent-duo. It is easy to see that $T=$ $K I+K a_{0}+K a_{1}+K a_{2}$ is a subring of $R$ ( $T$ is just the subalgebra generated by $a_{1}$ and $\left.a_{2}\right), a_{1} \in N(T)$ and $a_{1} a_{2}=a_{0} \notin a_{2} T=K a_{2}$. Hence $T$ is not right nilpotent-duo.

(ii) We take the ring $S=F(t)$ with the monomorphism $\sigma$ that is constructed in Example $1.5(1)$. Consider the skew polynomial ring $R_{0}=S[x ; \sigma]$ in which every element is of the form $\sum_{i=0}^{n} a_{i} x^{i}$, only subject to $x a=\sigma(a) x$ for $a \in S$. Then $R_{0}$ is a principal left ideal domain by the left-handed version of [21, Theorem 1.2.9(i, ii)]. So $R_{0}$ is a left Noetherian domain and hence $R_{0}$ has a left quotient ring which is a division ring by [21, Theorem 2.1.14], $Q\left(R_{0}\right)$ say.

Next consider $R_{1}=D_{2}\left(Q\left(R_{0}\right)\right)$. Then $R_{1}$ is nilpotent-duo by Lemma 1.8(3). To show that there exists a subring of $R_{1}$ which is not right nilpotent-duo, set $R=D_{2}\left(R_{0}\right)$. Then clearly $N(R)=\left(\begin{array}{cc}0 & R_{0} \\ 0 & 0\end{array}\right)$. Let

$$
A=\left(\begin{array}{ll}
x & 0 \\
0 & x
\end{array}\right) \in R \text { and } B=\left(\begin{array}{cc}
0 & t+x \\
0 & 0
\end{array}\right) \in N(R) .
$$

Then $B A=\left(\begin{array}{cc}0 & t x+x^{2} \\ 0 & 0\end{array}\right)$. Assume on the contrary that there exists $C \in N(R)$ such that $B A=A C$. Then $C=\left(\begin{array}{cc}0 & c(x) \\ 0 & 0\end{array}\right)$ for some $c(x) \in R_{0} . \quad A C=\left(\begin{array}{cc}0 & x c(x) \\ 0 & 0\end{array}\right)$ and $t x+x^{2}=x c(x)$ follows. Since $R_{0}$ is a domain, $c(x)=a_{0}+a_{1} x$ for some nonzero $a_{0}, a_{1} \in S$. It then follows that

$$
t x+x^{2}=x\left(a_{0}+a_{1} x\right)=\sigma\left(a_{0}\right) x+\sigma\left(a_{1}\right) x^{2},
$$


entailing $t=\sigma\left(a_{0}\right)$. But this equality is impossible because $\sigma\left(a_{0}\right)$ is of the form $\frac{f\left(t^{2 k}\right)}{g\left(t^{2 k}\right)}$. Therefore the subring $R$ of $R_{1}$ is not right nilpotent-duo.

(2) The class of right (left) nilpotent-duo rings is not closed under homomorphic images:

(i) Let $R$ be the ring of quaternions with integer coefficients. Then $R$ is a domain and so nilpotent-duo. However for any odd prime integer $q$, the $\operatorname{ring} R / q R$ is isomorphic to $\operatorname{Mat}_{2}\left(\mathbb{Z}_{q}\right)$ by the argument in $[10$, Exercise $2 \mathrm{~A}]$. But $\mathrm{Mat}_{2}\left(\mathbb{Z}_{q}\right)$ is not right (left) nilpotent-duo by $\operatorname{REMARKS}(1)$, and thus $R / q R$ is not right (left) nilpotent-duo.

(ii) Let $F$ be a field and $A=F\langle x, y\rangle$ be the free algebra with noncommuting indeterminates $x, y$ over $F$. Then $A$ is a domain and so nilpotent-duo. Consider $R=A / I$ with $I$ the ideal of $A$ generated by $x^{2}$. Then $R$ is neither left nor right nilpotent-duo as can be seen by $x y \notin y N(R)$ and $y x \notin N(R) y$, noting $x \in N(R)$. In fact, both $x y$ and $y x$ are nonzero; and letting $x y=y z$ (resp., $y x=z^{\prime} y$ ) with $z \in N(R)$ (resp., $z^{\prime} \in N(R)$ ), we get $0=x x y=x y z \neq 0$ (resp., $0=y x x=z^{\prime} y x \neq 0$ ); hence any case is impossible.

The following contains basic properties of one-sided nilpotent-duo rings.

Proposition 1.10. (1) Let $R_{\gamma}(\gamma \in \Gamma)$ be rings. Then $R_{\gamma}$ is a right (resp., left) nilpotentduo ring for each $\gamma \in \Gamma$ if and only if the direct sum $R=\bigoplus_{\gamma \in \Gamma} R_{\gamma}$ of rings $R_{\gamma}$ is a right (resp., left) nilpotent-duo ring.

(2) A ring $R$ is a right (resp., left) nilpotent-duo if and only if both $e R$ and $(1-e) R$ are right (resp., left) nilpotent-duo for any central idempotent e in $R$.

Proof. (1) Note that $N(R)=\bigoplus_{\gamma \in \Gamma} N\left(R_{\gamma}\right)$. Suppose that $R_{\gamma}$ is a right nilpotent-duo ring for $\gamma \in \Gamma$ and let $a=\left(a_{\gamma}\right)_{\gamma \in \Gamma} \in N(R)$. Then

$$
N(R) a=\bigoplus_{\gamma \in \Gamma}\left[N\left(R_{\gamma}\right) a_{\gamma}\right] \subseteq \bigoplus_{\gamma \in \Gamma}\left[a_{\gamma} N\left(R_{\gamma}\right)\right]=a N(R),
$$

by hypothesis. Hence the direct sum of rings $R_{\lambda}$ is right nilpotent-duo.

Conversely, suppose that $R=\bigoplus_{\gamma \in \Gamma} R_{\gamma}$ is right nilpotent-duo and let $a \in R_{\gamma}$, where $\gamma \in \Gamma$. Take $\alpha=\left(x_{\gamma}\right)_{\gamma \in \Gamma}$ such that $x_{\lambda}=a$ and $x_{\gamma}=0$ for all $\gamma \neq \lambda$ and $a=\left(a_{\gamma}\right)_{\gamma \in \Gamma}$ such that $a_{\lambda} \in N\left(R_{\lambda}\right)$ and $a_{\gamma}=0$ for all $\gamma \neq \lambda$. Then $a \alpha \in N(R) \alpha \subseteq \alpha N(R)$, noting that $R$ is right nilpotent-duo. This implies that $N\left(R_{\lambda}\right) a \subseteq a N\left(R_{\lambda}\right)$. Therefore $R_{\gamma}$ is right nilpotent-duo for each $\gamma \in \Gamma$. The proof for the left case is similar.

(2) Assume that $R$ is right nilpotent-duo. By Lemma 1.8(1), $R$ is abelian. So we have $N(e R)=e N(R)$ and $N((1-e) R)=(1-e) N(R)$. Using these facts, we can easily prove that both $e R$ and $(1-e) R$ are right nilpotent-duo.

The converse comes from $(1)$, since $R \cong e R \oplus(1-e) R$. The proof for the left case is similar.

As an application of Proposition 1.10(1), we show that the converse of Lemma 1.8(4) need not be true. In fact, consider the ring $T_{2}(R)$ over a right-nilpotent-duo ring $R$. Then $T_{2}(R)$ is not right nilpotent-duo by Remarks(1). For a nil ideal $I=\left(\begin{array}{ll}0 & R \\ 0 & 0\end{array}\right)$ of $T_{2}(R)$, $T_{2}(R) / I \cong R \oplus R$ is a right nilpotent-duo by Proposition 1.10(1).

Let $A$ be an algebra over a commutative ring $S$. Due to Dorroh [7], the Dorroh extension of $A$ by $S$ is the Abelian group $A \times S$ with multiplication given by

$$
\left(r_{1}, s_{1}\right)\left(r_{2}, s_{2}\right)=\left(r_{1} r_{2}+s_{1} r_{2}+s_{2} r_{1}, s_{1} s_{2}\right)
$$

for $r_{i} \in A$ and $s_{i} \in S$. We use $A \oplus_{\text {dor }} S$ to denote the Dorroh extension of $A$ by $S$.

Proposition 1.11. Let $R$ be a unitary algebra over a commutative reduced ring $S$. Then $R$ is right (resp., left) nilpotent-duo if and only if the Dorroh extension $R \oplus_{\text {dor }} S$ is right (resp., left) nilpotent-duo. 
Proof. Let $D=R \oplus_{\text {dor }} S$. First note that $s \in S$ is identified with $s 1 \in R$ and so $R=\{r+s \mid(r, s) \in D\}$, and that $N(D)=(N(R), 0)$ since $S$ is a commutative reduced ring.

Suppose that $R$ is right nilpotent-duo. Let $(r, s) \in D$ and $(n, 0) \in N(D)$. Then $(n, 0)(r, s)=(n r+s n, 0)=(n(r+s), 0) \in(N(R)(r+s), 0) \subseteq((r+s) N(R), 0)$ by hypothesis. There exists $n^{\prime} \in N(R)$ such that $n(r+s)=(r+s) n^{\prime}$, entailing that $(n, 0)(r, s)=$ $(r, s)\left(n^{\prime}, 0\right) \in(r, s) N(D)$. Thus $D$ is right nilpotent-duo.

Conversely, suppose that $D$ is right nilpotent-duo. Let $a \in R$ and $n \in N(R)$. Then $(n a, 0)=(n, 0)(a, 0) \in N(D)(a, 0) \subseteq(a, 0) N(D)$ by hypothesis. So there exists $n^{\prime} \in N(R)$ such that $(n a, 0)=(n, 0)(a, 0)=(a, 0)\left(n^{\prime}, 0\right)=\left(a n^{\prime}, 0\right)$ and thus $n a=a n^{\prime}$, showing that $N(R) a \subseteq a N(R)$. Therefore $R$ is right nilpotent-duo. The left case is similarly proved.

\section{Structure related to various sorts of rings}

In this section, we investigate structures of various kinds of rings in relation with nilpotent-duo property. We first observe some conditions under which the nilpotent-duo property can be left-right symmetric.

Proposition 2.1. (1) Let $R$ be a ring with an involution *. Then $R$ is left nilpotent-duo if and only if it is right nilpotent-duo.

(2) Let $K$ be a commutative ring and $G$ be a group. Then the group ring $K G$ is one-sided nilpotent-duo if and only if $K G$ is nilpotent-duo.

Proof. (1) Let $a \in N(R)$ and $b \in R$. Since $\left(a^{k}\right)^{*}=\left(a^{*}\right)^{k}$ for $k \geq 1$ and $0^{*}=0$, we have $a^{*} \in N(R)$. If $R$ is left nilpotent-duo, then $b^{*} a^{*}=c b^{*}$ for some $c \in N(R)$. This yields

$$
a b=\left((a b)^{*}\right)^{*}=\left(b^{*} a^{*}\right)^{*}=\left(c b^{*}\right)^{*}=b c^{*},
$$

noting that $c^{*} \in N(R)$. This leads us to conclude that $R$ is right nilpotent-duo. The proof of the converse is analogous.

(2) Consider the standard involution $*$ on $K G$, defined by $\left(\sum a_{i} g_{i}\right)^{*}=\sum a_{i} g_{i}^{-1}$ for all $a_{i} \in R$ and $g_{i} \in G$. Then $K G$ is left nilpotent-duo if and only if $K G$ is right nilpotent-duo, if and only if $K G$ is nilpotent-duo by (1).

If $G$ is an Abelian group and $K$ is a field then the group ring $K G$ is commutative, and hence is both duo and nilpotent-duo. In the next proposition we consider the case of non-Abelian groups.

Proposition 2.2. Let $K$ be a field of characteristic zero and $R$ be the group ring $K Q_{8}$, where $Q_{8}$ is the quaternion group. Then the following conditions are equivalent: (1) $R$ is reduced; (2) $R$ is nilpotent-duo; (3) $R$ is right (left) nilpotent-duo; (4) $R$ is abelian; (5) $R$ is IFP; (6) $R$ is right (left) duo; (7) $R$ is duo.

Proof. The proof is almost similar to ones of [2, Theorem 2.1] and [5, Proposition 1.7]. One-sided nilpotent-duo rings are abelian by Lemma 1.8(1), and reduced rings are clearly nilpotent-duo. Recall that the class of IFP rings contains one-sided duo rings and reduced rings, and that IFP rings are abelian. So it suffices to prove $(4) \Rightarrow(1)$ and $(4) \Rightarrow(7)$.

Note that $R$ is isomorphic to $K \oplus K \oplus K \oplus K \oplus H(K)$ such that $H(K)$ is either a division ring $D$ or $M_{a t}(K)$, by [24, Theorem 7.4.6 and Lemma 7.4.9]. Suppose that $R$ is abelian then $R$ is isomorphic to $K \oplus K \oplus K \oplus K \oplus D$. So $R$ is both duo and reduced, finishing the proof.

Following [9], a ring $R$ is said to be von Neumann regular if for each $a \in R$ there exists $b \in R$ such that $a=a b a$. We have the following proposition by Lemma 1.8(1), [9, Theorem 3.2 , and the definition of a nilpotent-duo ring. 
Proposition 2.3. For a von Neumann regular ring $R$, the following conditions are equivalent:

(1) $R$ is reduced; (2) $R$ is nilpotent-duo; (3) $R$ is right (left) nilpotent-duo; (4) $R$ is abelian; (5) $R$ is IFP; (6) $R$ is right (left) duo; (7) $R$ is duo.

Due to Nicholson [22], a ring is called an I-ring if every non-nil right ideal contains a nonzero idempotent. It is straightforward that the Jacobson radical of an I-ring is nil. Following Nicholson [23], a ring $R$ is said to be potent if idempotents can be lifted modulo $J(R)$ and every right (equivalently left) ideal not contained in $J(R)$ contains a nonzero idempotent. Note that I-rings are clearly potent, and potent rings with nil Jacobson radical are I-rings.

Proposition 2.4. (1) Let $R$ be a potent ring. Then we have the following results.

(i) If $R$ is abelian, then $N(R) \subseteq J(R)$ and $R / J(R)$ is reduced.

(ii) If $R$ is right (left) nilpotent-duo, then $N(R) \subseteq J(R)$ and $R / J(R)$ is reduced.

(2) Let $R$ be an I-ring. If $R$ is right (left) nilpotent-duo, then $N(R)=N^{*}(R)=J(R)$.

Proof. (1) (i) Suppose that $R$ is abelian. Let $a \in N(R)$ with $a^{n}=0$, and assume that $a R \nsubseteq J(R)$. Since $R$ is potent, there exists $0 \neq e^{2}=e \in R$ with $e \in a R, e=a b$ say. Since $e$ is central,

$$
e=e^{n}=(a b)^{n}=(a b)^{n-2} a b a b=(a b)^{n-2} a(a b) b=(a b)^{n-3} a a a b b b=\cdots=a^{n} b^{n}=0,
$$

a contradiction. Thus $a R \subseteq J(R)$ and $N(R) \subseteq J(R)$ follows.

Assume on the contrary that $c^{2} \in J(R)$ for some $c \notin J(R)$. Since $R$ is potent, there exists $0 \neq f^{2}=f \in c R, f=c d$ say. Since $f$ is central, $f=f^{2}=(c d)^{2}=c c d d \in J(R)$, a contradiction. Therefore $R / J(R)$ is reduced.

(ii) It follows from (1) and Lemma 1.8(1).

(2) Since $R$ is an I-ring, $J(R)$ is nil and $J(R)=N^{*}(R)$ follows. Moreover I-rings are potent, and so we get $N(R)=N^{*}(R)=J(R)$ by $(1)$.

Corollary 2.5 ([18, Lemma 3.1]). If $R$ a potent ring with $J(R)=0$, then $R$ is abelian if and only if $R$ is reduced.

The following elaborates on Proposition 2.4.

Example 2.6. There exists a potent and nilpotent-duo ring $R$ such that $N(R) \subsetneq J(R)$. Let $R_{0}=K[[x]]$ be the power series ring with an indeterminant $x$ over a field $K$. Next set $R=D_{2}\left(R_{0}\right)$. Then $R$ is nilpotent-duo by Theorem 1.2(5) because $R_{0}$ is commutative. Note that

$$
J(R)=\left\{\left(\begin{array}{ll}
f & g \\
0 & f
\end{array}\right) \mid f \in K[[x]] x \text { and } g \in R_{0}\right\},
$$

entailing that $R / J(R) \cong K$. It follows from this fact that idempotents can be lifted modulo $J(R)$. Thus $R$ is potent. But $J(R)$ is non-nil, and so $R$ is not an I-ring.

Following Marks [19], a ring $R$ is called $N I$ if $N(R)=N^{*}(R)$. Note that a ring $R$ is NI if and only if $N(R)$ forms an ideal of $R$ if and only if $R / N^{*}(R)$ is reduced. Notice that right nilpotent-duo rings are NI by Proposition 2.4(2) if they are I-rings. We consider other conditions under which right nilpotent-duo rings are NI.

Proposition 2.7. Let $R$ be a right (left) nilpotent-duo ring. Then we have the following.

(1) If $N(R)$ is nilpotent, then $R$ is $N I$.

(2) If $R$ is right Goldie, then $R$ is NI.

(3) If $R$ satisfies the ascending chain conditions on left and right annihilators, then $R$ is NI. 
Proof. (1) Assume that $N(R)$ is nilpotent. Say $N(R)^{k}=0$ for some $k \geq 1$. Let $a \in N(R)$ and $r \in R$. We use the right nilpotent-duo property of $R$ freely to prove $a r \in N(R)$. Consider $(a r)^{k}$. Then $a r=r a_{1}$ for some $a_{1} \in N(R)$. By the same argument as in the proof of Theorem 1.1(1), we obtain $a_{i} r=r a_{i+1}$ with $a_{i} \in N(R)$ for all $i \geq 1$, and so $(a r)^{k}=r^{k} a_{k} a_{k-1} \cdots a_{2} a_{1}=0$, from the fact of $N(R)^{k}=0$. This implies $a R$ is nil, and $a R \subseteq N^{*}(R)$ follows because Köthe's conjecture holds for $R$ by Theorem 1.1(2). Therefore $R$ is NI.

(2) Since $R$ is right nilpotent-duo, $N(R)$ is a nil subring of $R$ by Theorem 1.1(1). If $R$ is right Goldie, then $N(R)$ is nilpotent by [17]. Thus the proof is completed by (1).

(3) Suppose that $R$ satisfies the ascending chain conditions on left and right annihilators. Then any nil subring of $R$ is nilpotent by [11, Theorem 1]. So (1) and Theorem 1.1(1) can be applied to this case.

By the argument prior to [12, Theorem 2], there exist nil rings, satisfying the ascending chain condition on left annihilators, which are not nilpotent. Next we observe the property of annihilators in $N(R)$ of a right (left) nilpotent-duo ring $R$.

Let $R$ be a ring and $S \subseteq R$. The left (resp., right) annihilator of $S$ in $R$ is denoted by $l_{R}(S)$ (resp., $r_{R}(S)$ ).

Proposition 2.8. (1) Let $R$ be a one-sided nilpotent-duo ring which satisfies the ascending chain condition on left (resp., right) annihilators. Then $r_{N(R)}(N(R))\left(\right.$ resp., $\left.l_{N(R)}(N(R))\right)$ is nonzero when $N(R) \neq 0$.

(2) Let $R$ be a one-sided nilpotent-duo ring with an involution, which satisfies the ascending chain condition on left or right annihilators. Then $N(R)$ is nilpotent and $R$ is NI.

Proof. (1) Note that $N(R)$ is a nil subring of $R$ by Theorem 1.1(1). By hypothesis, $N(R)$ also satisfies the ascending chain condition on left annihilators, and so $r_{N(R)}(N(R)) \neq 0$ by [11, Lemma 2]. For the proof of the case that $R$ satisfies the ascending chain condition on right annihilators, we refer to $[4, \operatorname{Remarks}(2)$, page 33$]$

(2) By Proposition 2.1(1), one-sided nilpotent-duo rings are nilpotent-duo because $R$ has an involution. So $N(R) a=a N(R)$ for all $a \in R$; hence $N(R) a=0$ implies $a N(R)=0$ and vice versa. Thus every left annihilator of $N(R)$ in $R$ (hence in $N(R)$ ) is also right annihilator, and vice versa.

Assume that $R$ satisfies the ascending chain condition on left annihilators. Then $N(R)$ satisfies the ascending chain condition on left annihilators. So by the preceding argument, $N(R)$ also satisfies the ascending chain condition on right annihilators. The proof, with the assumption that $R$ satisfies the ascending chain condition on right annihilators, is similar. This finishes the proof by help of Proposition 2.7(3).

Following Yao [26], a ring $R$ is called weakly right (resp., left) duo if for each $a \in R$ there exists $n \geq 1$, depending on $a$, such that $a^{n} R$ (resp., $R a^{n}$ ) is two-sided. Weakly right duo rings are abelian by [26, Lemma 4]. Right duo rings are obviously weakly right duo, but the converse does not hold in general by considering $E=D_{n}(R)$ over a division ring $R$ for $n \geq 3$. Indeed, every matrix in $E$ is either a unit or a nilpotent, but $E_{23} E$ is not two-sided.

Recall that a ring is called locally finite [14] if every finite subset generates a finite multiplicative semigroup. Finite rings are clearly locally finite, and there exist locally finite rings but not finite (e.g., algebraic closures of finite fields). Note that $E=D_{n}(R)$, over a locally finite abelian ring $R$ for $n \geq 3$, is weakly right duo by [13, Lemma 2] and the proof of [14, Proposition 16]. However $E$ is not right nilpotent-duo by Example 1.4. 
Proposition 2.9. Let $R$ be a right (resp., left) nilpotent-duo ring. Then we have the following.

(1) If $R$ is a weakly right (resp., left) duo ring then $R$ is NI.

(2) If $R$ is a locally finite ring then $R$ is NI.

Proof. (1) Suppose that $R$ is a weakly right duo ring. Then for any $a \in N(R), a R$ is nil by [26, Lemma 2]. Moreover $a R$ is contained in $N^{*}(R)$ by Theorem 1.1(2). Thus $N(R)=N^{*}(R)$. The proof for the left case is similar.

(2) Note first that $R$ is abelian by Lemma 1.8(1) because $R$ is right nilpotent-duo. Let $R$ be a locally finite ring. Then $R$ is weakly right duo by [16, Proposition 15]. So $R$ is NI by (1). The left case is similarly proved.

Notice that ' $a R$ is contained in $N^{*}(R)$ ' in the second part of the proof of Proposition 2.9(1) is true if $N(R) \subseteq Z(R)$, where $Z(R)$ denotes the center of a ring $R$. But there exists a right nilpotent-duo ring $R$ such that $N(R) \nsubseteq Z(R)$, for example, the ring $R$ in the proof of Theorem 2.10(2) to follow. Indeed, $N(R)=\left(\begin{array}{ll}0 & D \\ 0 & 0\end{array}\right)$ and $Z(R)=$ $\left\{\left(\begin{array}{ll}c & d \\ 0 & c\end{array}\right) \mid c, d \in Z(D)\right\}$, so that $N(R) \nsubseteq Z(R)$ because $Z(D) \subsetneq D$.

Next we show that the right nilpotent-duo property does not go up to polynomial rings

Theorem 2.10. (1) Let $R$ be a ring with $N(R) \neq 0$. Suppose that the polynomial ring $R[x]$ is right (resp., left) nilpotent-duo. Then $a b=b a$ for all $a \in R$ and $b \in N(R)$. Moreover $R$ is nilpotent-duo, $N(R)$ is a commutative ring, and $R[x]$ is an NI ring.

(2) There exists a right (resp., left) nilpotent-duo ring over which the polynomial ring is not right (resp., left) nilpotent-duo.

Proof. (1) Suppose that $R[x]$ is right nilpotent-duo. Let $a \in R$ and $b \in N(R)$. Then $b(a+x)=(a+x)\left(b_{0}+b_{1} x+\cdots+b_{n} x^{n}\right)$ for some $b_{0}+b_{1} x+\cdots+b_{n} x^{n} \in N(R[x])$ because $R[x]$ is right nilpotent-duo. But, checking the degrees of both sides of the equality, $n$ must be zero, i.e., $b_{0}+b_{1} x+\cdots+b_{n} x^{n}=b_{0}$. It then follows that $b_{0} \in N(R), b a=a b_{0}$, and $b=b_{0}$. Thus $b a=a b$. This implies that $R$ is nilpotent-duo, and $N(R)$ is a commutative ring by help of Theorem 1.1(1).

Next we show that $R[x]$ is an NI ring. Note first that $N(R)[x]$ is a commutative nil ring. Let $r \in R$ and $a \in N(R)$. Then $a r, r a \in N(R)$ because $a r=r a$ by the argument above. This implies $N(R)=N^{*}(R)$ (i.e., $R$ is an NI ring). It then follows that $N(R[x]) \subseteq N(R)[x]$ via the fact that $R[x] / N(R)[x] \cong(R / N(R))[x]$ and $R / N(R)$ is a reduced ring. This entails $N(R[x])=N(R)[x]$ because $N(R)[x]$ is nil. Let $f(x) \in R[x]$ and $g(x) \in N(R[x])$. Then $g(x) \in N(R)[x]$, and $f(x) g(x)=g(x) f(x)$ follows because $a b=b a$ for all $a \in R$ and $b \in N(R)$. This yields that $N(R[x])$ is an ideal of $R[x]$, and $R[x]$ is NI. The left case is similarly proved.

(2) Let $R=D_{2}(D)$ where $D$ is a noncommutative reduced right duo ring (e.g., direct products of noncommutative division rings). Then $R$ is right nilpotent-duo by Theorem 1.2(5). Assume that $a b \neq b a$ for some $a, b \in D$. Consider two matrices

$$
A=\left(\begin{array}{ll}
a & 0 \\
0 & a
\end{array}\right) \in R \text { and } B=\left(\begin{array}{ll}
0 & b \\
0 & 0
\end{array}\right) \in N(R) .
$$

Then $A B=\left(\begin{array}{cc}0 & a b \\ 0 & 0\end{array}\right) \neq\left(\begin{array}{cc}0 & b a \\ 0 & 0\end{array}\right)=B A$. So $R[x]$ is not right nilpotent-duo by (1). The proof for the left case is similar.

From Theorem 1.2(4) and Theorem 2.10, we obtain the following. 
Corollary 2.11. For a ring $R$, the following conditions are equivalent:

(1) $D_{2}(R)[x]$ is right (resp., left) nilpotent-duo;

(2) $R$ is commutative;

(3) $D_{2}(R)$ is commutative;

(4) $D_{2}(R)[x]$ is commutative.

Proof. It suffices to prove (1) implying (2). Suppose that $D_{2}(R)[x]$ is right nilpotentduo, and let $a, b \in R$. Consider $A=\left(\begin{array}{ll}a & 0 \\ 0 & a\end{array}\right) \in D_{2}(R)$ and $B=\left(\begin{array}{ll}0 & b \\ 0 & 0\end{array}\right) \in N\left(D_{2}(R)\right)$. By Theorem 2.10(1), $\left(\begin{array}{cc}0 & a b \\ 0 & 0\end{array}\right)=A B=B A=\left(\begin{array}{cc}0 & b a \\ 0 & 0\end{array}\right)$ and $a b=b a$ follows. The left case is similarly proved.

Acknowledgment. The authors thank the referees deeply for very careful reading of the manuscript and valuable suggestions in depth that improved the paper by much. The third and fourth authors were supported by the National Research Foundation of Korea(NRF) grant funded by the Korea government(MSIT) (No.2019R1F1A1057940) and (No.2019R1F1A1040405), respectively.

\section{References}

[1] H.E. Bell, Near-rings in which each element is a power of itself, Bull. Austral. Math. Soc. 2, 363-368, 1970.

[2] H.E. Bell and Y. Li, Duo group rings, J. Pure Appl. Algebra, 209, 833-838, 2007.

[3] H.H. Brungs, Three questions on duo rings, Pacific J. Math. 58, 345-349, 1975.

[4] A.W. Chatters and C.R. Hajarnavis, Rings with Chain Conditions, Pitman Advanced Publishing Program, Boston, London, Melbourne, 1980.

[5] Y.W. Chung and Y. Lee, Structures concerning group of units, J. Korean Math. Soc. 54, 177-191, 2017.

[6] R.C. Courter, Finite dimensional right duo algebras are duo, Proc. Amer. Math. Soc. 84, 157-161, 1982.

[7] J.L. Dorroh, Concerning adjunctions to algebras, Bull. Amer. Math. Soc. 38, 85-88, 1932.

[8] E.H. Feller, Properties of primary noncommutative rings, Trans. Amer. Math. Soc. 89, 79-91, 1958.

[9] K.R. Goodearl, Von Neumann Regular Rings, Pitman, London, 1979.

[10] K.R. Goodearl and R.B. Warfield, Jr., An Introduction to Noncommutative Noetherian Rings, London Mathematical Society Student Texts 16, Cambridge University Press, Cambridge, 1989.

[11] I.N. Herstein and L.W. Small, Nil rings satisfying certain chain conditions, Canad. J. Math. 16, 771-776, 1964.

[12] I.N. Herstein and L.W. Small, Addendum to "Nil rings satisfying certain chain conditions", Canad. J. Math. 18, 300-302, 1966.

[13] C. Huh, H.K. Kim and Y. Lee, p.p. rings and generalized p.p. rings, J. Pure Appl. Algebra, 16, 37-52, 2002.

[14] C. Huh, Y. Lee and A. Smoktunowicz, Armendariz rings and semicommutative ring, Comm. Algebra, 30, 751-761, 2002.

[15] S.U. Hwang, Y.C. Jeon and Y. Lee, Structure and topological conditions of NI rings, J. Algebra , 302, 186-199, 2006.

[16] H.K. Kim, N.K. Kim and Y. Lee, Weakly duo rings with nil Jacobson radical, J. Korean Math. Soc. 42, 455-468, 2005.

[17] C. Lanski, Nil subrings of Goldie rings are nilpotent, Canad. J. Math. 21, 904-907, 1969. 
[18] B. Li, On potent rings, Commun. Korean Math. Soc. 23, 161-167, 2008.

[19] G. Marks, On 2-primal Ore extensions, Comm. Algebra, 29, 2113-2123, 2001.

[20] G. Marks, Duo rings and Ore extensions, J. Algebra, 280, 463-471, 2004.

[21] J.C. McConnell, J.C. Robson, Noncommutative Noetherian Rings, John Wiley \& Sons Ltd., Chichester, New York, Brisbane, Toronto, Singapore, 1987.

[22] W.K. Nicholson, I-rings, Trans. Amer. Math. Soc. 207, 361-373, 1975.

[23] W.K. Nicholson, Lifting idempotents and exchange rings, Trans. Amer. Math. Soc. 229 , 269-278, 1977.

[24] C. Polcino Milies and S.K. Sehgal, An Introduction to Group Rings, Kluwer Academic Publishers, Dordrecht, 2002.

[25] G. Thierrin, On duo rings, Canad. Math. Bull. 3, 167-172, 1960.

[26] X. Yao, Weakly right duo rings, Pure Appl. Math. Sci. 21, 19-24, 1985. 